\title{
LETTERS
}

doi:10.1017/\$1041610214001793

\section{Does past or present depression differentiate Lewy body from Alzheimer disease?}

Clinical differentiation of Lewy body disease (LBD) from Alzheimer disease (AD) is still problematic. Many persons with LBD lack the cardinal features of visual hallucinations, fluctuations in cognition, and mild Parkinsonism proposed by McKeith et al. (2005). Some studies suggest that history or presence of depression may help distinguish $\mathrm{LBD}$ from $\mathrm{AD}$, but this is confounded because many clinically diagnosed LBD patients have significant co-morbid $\mathrm{AD}$ pathology and vice versa (Ranginwala et al., 2008). We aimed to clarify whether history or symptoms of depression differentiate $\mathrm{LBD}$ from $\mathrm{AD}$, in autopsy-confirmed patients, excluding patients with mixed $\mathrm{AD}$ and LBD pathology.

We examined clinical and pathological characteristics from the Alzheimer's Disease Center (ADC) database at the University of Texas (UT) Southwestern Medical Center collected between 1986 and 2011. The sample is composed of consecutive persons seen at the UT Southwestern ADC who had consented antemortem to autopsy or whose families sought postmortem confirmation of clinical diagnosis. All participants had consented for their data to be used on forms approved by the UT Southwestern Institutional Review Board.

Pathological diagnosis of $\mathrm{AD}$ was made with thioflavin-S stains for plaques and tangles. Neocortical Lewy bodies were detected by immunostaining for alpha synuclein. Our AD patients had essentially no neocortical Lewy bodies; LBD patients had only rare neuritic plaques and neurofibrillary tangles.

We used loose criteria for depression to reduce false negatives. Prevalent depression at initial visit was established by self report or observation of depressed mood by a clinician. Incident depression was diagnosed if either of the preceding criteria was met on any follow-up visit. Lifetime prevalence was defined as meeting the above criteria for depression on any visit, or report of a previous depression.

Depression and autopsy data were available for $195 \mathrm{AD}$ and $20 \mathrm{LBD}$ patients. There was a gender difference between groups $\left(\chi^{2}=5.733\right.$, $\mathrm{df}=1$, $p=0.017$ ), with LBD participants more likely to be male $(70 \%$ vs. $42 \%)$. The two groups did not differ in education, $(M=13.80, \mathrm{SD}=3.04$ vs.
$M=13.66, \mathrm{SD}=3.08$ years $)$, age at death $(M=$ $79.82, \mathrm{SD}=9.15$ vs. $M=78.18, \mathrm{SD}=9.48$ years $)$, at initial visit $(M=74.91, \mathrm{SD}=7.86$ vs. $M=$ $72.70, \mathrm{SD}=9.59$ years), listed respectively. The two groups had a median of two visits, ranging from 1 to 19 visits for $\mathrm{AD}$ (with a range of $<1$ to 15 years from initial visit to death) and 1 to 22 visits for LBD patients (ranging from 1 to 13 years from first visit to death). Although data for depression were obtained from any visit, only visits that included cognitive testing are reported here.

We found an initial prevalence of depression in $5.0 \%(1 / 20)$ of the LBD and $6.6 \%(13 / 195)$ of the $\mathrm{AD}$ sample (Fisher's Exact $p=0.774$ ), and in $6.9 \%$ of combined LBD and AD patients.

Although groups did not differ significantly in initial prevalence of depression, incident depression was significantly higher among the LBD group; $20.0 \%(4 / 20)$ vs. $2.6 \%$ (5/195) for the AD group (Fisher's Exact $p=0.005$ ). The lifetime prevalence of depression also differed significantly between groups; $25 \%$ (5/20) for the LBD and 9.2\% (18/195) for the $\mathrm{AD}$ group $\left(\chi^{2}=3.75, p=0.030\right)$.

Both incidence and lifetime prevalence predicted autopsy-confirmed LBD. In separate logistic regression models including sex, age at initial visit, and education (using the criterion of $p<0.15$ ), the lifetime prevalence of depression was 3.54 times higher $(95 \% \mathrm{CI}=1.04$ to $11.98, p=0.043)$ in the LBD group. Incident depression was 11.76 times ( $95 \% \mathrm{CI}=2.58$ to $53.57, p=0.001)$ higher in the LBD group and the initial prevalence of depression was non-significant ( $p=0.568)$.

Our findings support investigations finding a difference in depression incidence between LBD and AD patients (Klatka et al., 1996; Boot et al., 2013). The difference between our findings and studies showing higher rates of depression in LBD may partly be due to methodological issues in assessing depression in demented patients, but we deliberately set a low threshold for the diagnosis of depression. No formal assessment of depressed mood was conducted in this study, which relied on self report and clinical observation of depression. Though this approach has limitations, such as the potential to inflate depression prevalence and incidence rates, this did not appear to occur in the current investigation. A limitation of our study is the small sample size while a strength is that we examined differences between "pure" $\mathrm{AD}$ and LBD patients. Because we used a convenience sample, participants may not represent 
the general population. Another possible weakness in our study is the use of retrospective self-report, possibly decreasing the report of lifetime depression prevalence.

In conclusion, we found a significant difference of incidence and lifetime prevalence of depression in autopsy-diagnosed LBD and AD patients and a preponderance of men in the LBD group. Thus, a history of past depressive episodes and the development of depression after the initial visit in a male should raise the suspicion of LBD alone or as a co-morbidity.

\section{Conflict of interest}

None.

\section{Acknowledgments}

This study was supported by NIA 5P30 AG012300, the Winspear Family Center for Research on the Neuropathology of Alzheimer's Disease, and the McCune Foundation.

\section{References}

Boot, B. P. et al. (2013). Risk factors for dementia with Lewy bodies: a case-control study. Neurology, 81, 833-840.

doi:10.1017/\$1041610214002518

\section{Late-onset psychosis in older outpatients: a retrospective chart review}

Late-onset psychosis (LOP) has become increasingly prevalent in the clinical setting, especially in the highly aged society, due to the increasing numbers of older people and its disruptive impact on the lives of patients and caregivers. Although previous studies have identified some of the features of LOP (Webster and Grossberg, 1998; Mitford et al., 2010; Tan and Seng, 2012), some of the previous studies did not include patients with dementia and mood disorders. This study addresses the features of LOP in consecutive outpatients to provide information that supports the differential diagnosis.

We enrolled 1,024 consecutive outpatients over the age of 60 years who were seen at the department of Psychiatry, Kyoto Prefectural University of Medicine, between April 2009 and March 2013. A retrospective chart review of 1,024 outpatients was conducted. The outpatients with LOP were defined
Klatka, L. A., Louis, E. D. and Schiffer, R. B. (1996). Psychiatric features in diffuse Lewy body disease: a clinicopathologic study using Alzheimer's disease and Parkinson's disease comparison groups. Neurology, 47, 1148-1152.

McKeith, I. G. et al. (2005). Diagnosis and management of dementia with Lewy bodies third report of the DLB consortium. Neurology, 65, 1863-1872.

Ranginwala, N. A. et al. (2008). Clinical criteria for the diagnosis of Alzheimer disease: still good after all these years. The American fournal of Geriatric Psychiatry, 16, 384-388.

JED FALKOWSKI, ${ }^{1}$ LINDA S. HYNAN, ${ }^{1,2,3}$
KYLE B. WOMACK, ${ }^{3,4}$
KIMMO J. HATANPAA, ${ }^{3,5}$
CHARLES L. WHITE III ${ }^{3,5}$
AND MYRON F. WEINER ${ }^{1}$
${ }^{1}$ Department of Psychiatry, University of Texas
Southwestern Medical Center, Dallas, Texas, USA
Email: JAF14@alumni.utsw.edu
${ }^{2}$ Department of Clinical Sciences, Division of
Biostatistics, University of Texas Southwestern
Medical Center, Dallas, Texas, USA
${ }^{3}$ Alzheimer's Disease Center, University of Texas
Southwestern Medical Center, Dallas, Texas, USA
${ }^{4}$ Department of Neurology and Neurotherapeutics,
University of Texas Southwestern Medical Center,
Dallas, Texas, USA
${ }^{5}$ Department of Pathology, University of Texas
Southwestern Medical Center, Dallas, Texas, USA

as the outpatients who had first manifestations of psychosis, including delusions and hallucinations, at the age of 60 years or older. The early-onset psychosis (EOP) was defined as the first psychosis manifestation below the age of 60 years. The diagnosis was made by psychiatrists, according to the ICD-10. We defined hearing or visual impairment as poor hearing or visual capacity in the clinical examination and daily life. Thought disorder included thought broadcasting, loosening of associations, and blocking of thought, which was usually found in schizophrenia. Negative symptoms included affective flattening and impoverishment of speech. All data were coded and registered anonymously. We used the $\chi^{2}$ test for the statistical analyses. In post hoc analyses, we used the $\chi^{2}$ test with pairwise comparison. Differences in age were compared using one-way ANOVA with Bonferroni post hoc test. For the logistic regression analysis, a forward selection method (likelihood ratio) was used to determine the predictors of LOP. Variables, including age, gender, living alone, hearing impairment, visual impairment, and family 